Review Article

\title{
Clinical Randomized Controlled Study of Acupuncture Treatment on Children with Autism Spectrum Disorder (ASD): A Systematic Review and Meta-Analysis
}

\author{
Lei Wang, ${ }^{1}$ Jin-Lin Peng, ${ }^{2}$ Fu-Qiang Qiao, ${ }^{3}$ Wen-Ming Cheng, ${ }^{4}$ Guang-Wen Lin, ${ }^{4}$ \\ Yao Zhang, ${ }^{3}$ Tian-Ge Gao, ${ }^{3}$ Ying-Ying Sun, ${ }^{3}$ Wei-Zhong Tang, ${ }^{4}$ and Pu Wang ${ }^{1}$ \\ ${ }^{1}$ Department of Rehabilitation Medicine, The Seventh Affiliated Hospital, Sun Yat-Sen University, Shenzhen 518107, \\ Guangdong, China \\ ${ }^{2}$ Tongji Hospital, Tongji Medical College, Huazhong University of Science \& Technology, Wuhan 430000, Hubei, China \\ ${ }^{3}$ School of Education and Psychology, University of Jinan, Jinan 250000, Shandong, China \\ ${ }^{4}$ Department of Wei Zhong Children's Rehabilitation Center, Jinan 250000, Shandong, China
}

Correspondence should be addressed to Pu Wang; wangpu183@163.com

Received 18 February 2021; Revised 31 May 2021; Accepted 6 July 2021; Published 26 July 2021

Academic Editor: Longfei Yang

Copyright (c) 2021 Lei Wang et al. This is an open access article distributed under the Creative Commons Attribution License, which permits unrestricted use, distribution, and reproduction in any medium, provided the original work is properly cited.

\begin{abstract}
This study aimed to summarize the effectiveness and safety of acupuncture in the treatment of autism spectrum disorder (ASD) through literature analysis and evaluation. All studies were retrieved from various databases as follows: English databases, such as PubMed, Cochrane Library, Ovid, and Web of Science, and Chinese databases, such as China National Knowledge Infrastructure (CNKI), WanFang Data (WF), and Technology Periodical Database (VIP). The Cochrane Collaboration's Bias Risk Assessment Scale was used to assess the studies' risk of bias. The effects of acupuncture treatment for ASD were determined using the following indicators: childhood autism rating scale (CARS), autism behavior check list (ABC), Reynell developmental language scale (RDLS), and functional independence measure of children (WeeFIM). The risk map of bias of these studies' quality and the metaanalysis results of the indicators was prepared with RevMan 5.2 software. Finally, 16 studies were included, five of which were in English and 11 were in Chinese. The 16 studies included 1332 patients. The CARS results for subgroup analysis were as follows: acupuncture subgroup $(\mathrm{MD}=-2.65,95 \% \mathrm{CI}(-3.22,-2.07))$ and acupuncture plus massage subgroup $(\mathrm{MD}=-10.35,95 \% \mathrm{CI}$ $(-11.34,-9.36))$. The ABC results were as follows: $(\mathrm{MD}=-6.70,95 \% \mathrm{CI}(-9.10,-4.29))$. The analysis results of sensory, relating, language, body and object use, and social/self-help in the subitems of $\mathrm{ABC}$ were as follows: sensory $(\mathrm{MD}=-2.67,95 \% \mathrm{CI}(-2.90$, $-2.44)$ ), relating $(\mathrm{MD}=-3.28,95 \% \mathrm{CI}(-3.55,-3.02))$, language $(\mathrm{MD}=-2.45,95 \% \mathrm{CI}(-2.73,-2.16))$, body and object use $(\mathrm{MD}=-1.19,95 \% \mathrm{CI}(-1.38,-1.00))$, and social/self-help $(\mathrm{MD}=-2.09,95 \% \mathrm{CI}(-2.30,-1.89))$. For the analysis results of comprehension and expression ages in the subitems of RDLS, the comprehension age results were as follows: (MD $=0.08,95 \% \mathrm{CI}$ $(-0.06,0.22), P=0.27)$. Those of expression age were as follows: $(\mathrm{MD}=0.15,95 \% \mathrm{CI}(0.04,0.26), P=0.009)$. The WeeFIM results were as follows: $(\mathrm{MD}=3.70,95 \% \mathrm{CI}(2.38,5.02))$. This study suggested that acupuncture could effectively treat ASD. However, acupuncture methods and prescriptions at this stage remain heterogeneous, and acupuncture treatment operations require standardization. Studies using rigorous and standard research designs are needed to draw stronger conclusions about the advantages of using acupuncture to treat children and adolescents with ASD.
\end{abstract}

\section{Introduction}

Autism spectrum disorder (ASD), also known as autism, is a type of mental development disorder that starts before the age of 3 years old. ASD is characterized by social communication disorders, limitations, stereotypes, and repetitive behaviors. ASD is now categorized as a mental illness $[1,2]$. In epidemiological surveys, the median global prevalence of ASD was 62 in 10,000 [3]. According to the Second Epidemiological Sample Survey of the Disabled, in 
China, approximately 41,000 children aged 0-6 years old have ASD [1]. According to the report of the World Health Organization (WHO) in 2017, approximately 1 in 160 children suffers from ASD, and this proportion is still increasing [4]. ASD is accompanied by various problems, such as abnormal emotions, abnormal behaviors, and developmental disorders. Children with ASD usually incur longterm social and family medical costs. In the United States, the estimated total annual cost of children with ASD is between $\$ 11.5$ billion and $\$ 60.9$ billion [5]. The medical expenses of children and adolescents with ASD are 4.1-6.2 times those of children and adolescents without ASD [6].

Improving the curative effect of treatments of patients with ASD has become a long-term concern of the clinical medical staff. Nowadays, the commonly used clinical interventions for ASD include behavioral interventions and drugs. Behavioral interventions are more common than drugs [7]. However, behavioral interventions require large-scale venues and specialized facilities, one-to-one treatment, and the help of professionals trained in institutions for long-term work; these requirements consume human resources and are expensive [8]. The effectiveness of behavioral interventions is limited [9], and it is usually combined with other approaches, such as drugs [10]. Drug treatment also has limitations. The current drugs used for treating ASD are only helpful for a small number of people. The US Food and Drug Administration (FDA) has approved very few drugs for the treatment of ASD [11]. Drugs serve only to reduce irritability and hyperactivity behavior and are not effective in reducing social and language barriers for most patients with ASD [12]. In addition, long-term drug use results in side effects, such as weight gain, fatigue, drowsiness, and tremors, for example, risperidone treatment $[13,14]$.

Considering the abovementioned reasons, researchers are focusing more on the role of complementary and alternative medicine in ASD. In the United States, complementary and alternative medicines used to treat ASD involve diet modifications and food supplements [10]. Acupuncture is rarely used as an intervention for children with ASD in the United States [15]. However, in China and other Asian countries, acupuncture is widely used in the treatment with ASD. In a survey conducted in Hong Kong, acupuncture has reportedly been used on approximately $40 \%$ of children with ASD; it has become the most common form of complementary and alternative medicine used for this condition [16]. In China, acupuncture and other TCM methods are considered alternative therapies that need to be verified in accordance with the guidelines for the diagnosis, treatment, and rehabilitation of children with autism issued by the Ministry of Health in 2010 [1]. The treatment of ASD with acupuncture can be performed in many ways, including electroacupuncture [17], tongue acupuncture [18, 19], scalp acupuncture [20,21], and total body acupuncture [10].

In this study, a meta-analysis of clinical randomised controlled studies was performed by searching relevant articles. The aims of this study were as follows: to evaluate acupuncture's efficacy and safety in the treatment of children and adolescents with ASD, to further search for stronger evidence that supports the use of acupuncture as a treatment for ASD, and to analyze the deficiencies of current studies.

\section{Methods}

2.1. Study Search. All studies were retrieved from various databases as follows: English databases, such as PubMed, Cochrane Library, Ovid, and Web of Science, and Chinese databases, such as China National Knowledge infrastructure (CNKI), WanFang Data (WF), and Technology Periodical Database (VIP). Articles published from the establishment of the database to October 2020 were included in the search. The articles must either be in Chinese or in English. We performed several presearches based on related subject words and free words. We determined the final search formula and collected articles based on this formula. The search terms were as follows: acupuncture, scalp acupuncture, electroacupuncture, tongue acupuncture, traditional Chinese medicine (TCM), autism spectrum disorders, ASDs, ASD, and autism. Two independent authors (Lei Wang and Jin-lin Peng) conducted a literature search according to the search strategy. The disputed part was addressed by a third author (Pu Wang).

2.2. Inclusion and Exclusion Criteria. The inclusion criteria were as follows: (a) participants were diagnosed with ASD, (b) the intervention method involved an experimental group subjected to acupuncture, and (c) the study type was a randomised controlled one. The exclusion criteria were as follows: (a) the conventional treatment in the intervention measures did not meet the treatment recommended by the guidelines, (b) reviews and repeated articles, (c) the full text being unavailable or articles having incomplete data, and (d) animal studies.

2.3. Data Extraction. We extracted the following data: (a) normal information including first author, year of publication, sample size, acupoint, age, gender, intervention measures, and treatment course; (b) outcome indicators, including childhood autism rating scale (CARS), autism behavior check list $(A B C)$, subitems of $A B C$, Reynell developmental language scale (RDLS), subitems of RDLS, and functional independence measure of children (WeeFIM). Two independent authors (Lei Wang and Jin-lin Peng) conducted a literature search according to the search strategy. The disputed part was addressed by a third author (Pu Wang).

2.4. Quality Evaluation. The included studies' quality was evaluated by two independent authors (Lei Wang and Jin-lin Peng). If a disagreement arose during the review process, a third author made the decision. The Cochrane Collaboration's Bias Risk Assessment Scale was used to assess the studies' risk of bias. Each study was assessed for selection bias (random sequence generation and allocation concealment), performance bias (blinding of participants and personnel), detection bias (blinding of outcome assessment), attrition bias (incomplete outcome data reporting), and reporting bias (selective outcome reporting). Each domain was rated as follows: high risk of bias, unclear bias, or low 
risk of bias [22]. A risk map of bias of these studies' quality was prepared by using RevMan 5.2 software. GRADEpro GDT online tool was used to assess the quality of evidence. The tool is available at the official website: http://www. guidelinedevelopment.org/.

2.5. Statistical Analysis. The Review Manager 5.2 software of Cochrane Collaboration was used for the meta-analysis. The outcome variables were continuous. Thus, the mean difference (MD) was calculated for the results, and the 95\% CI of the statistical results was reported. The chi-square test was used to calculate the heterogeneity of the included literature. When heterogeneity was $P>0.1$ and $I^{2}<50 \%$, a fixed-effect model was used. When the heterogeneity was $I^{2}>50 \%$, the causes of heterogeneity were analyzed through a subgroup or sensitivity analysis. If the results still had heterogeneity, then the random-effect mode was used for summary analysis [22]. If the number of included studies was sufficient $(n \geq 10)$, then the funnel chart was used to perform a bias analysis.

\section{Results}

3.1. Search Results. A total of 444 studies were retrieved, and all were imported into the Document Management Software of "Medical Literature King V6." A total of 182 duplicate studies were eliminated by using the function of duplication removal, 157 studies were excluded after reading the title and abstract, and 9 studies were excluded after reading the original text. Finally, 16 studies were included [17-21, 23-33], as shown in Figure 1.

3.2. Risk of Bias. The results of Cochrane Collaborative Network Bias Risk Assessment Scale Evaluation are shown in Figures 2 and 3. Twelve studies reported the source of random sequences, and five explained the implementation of allocation-hiding schemes [17-19, 30, 33]. Guaranteeing the blinding method in acupuncture and rehabilitation measures was difficult. Thus, only two studies were included to ensure the double-blinding of the experiment. The two groups were provided control groups to implement the method of sham acupuncture for double-blinding [17, 19]. Five studies reported that the evaluation of experimental results was blinded [17-19, 26, 29], whereas one study reported that the evaluation of the outcome was not blinded [21]. Two studies had insufficient data integrity and did not report and analyze the data on patients lost to follow-up and those who withdrew from the study $[18,30]$.

3.3. Quality of Evidence. The study included a total of 12 outcome indicators. The GRADEpro GDT online tool was used to assess the quality of evidence. Only the acupuncture subgroup of CARS showed moderate quality. CARS showed low quality, and the other indicators showed very low quality. The results are shown in Table 1.

3.4. Study Characteristics. As shown in Table 2, the characteristics included in the studies were as follows: first author, year of publication, sample size, acupoint, age, gender, intervention measures, and treatment course.

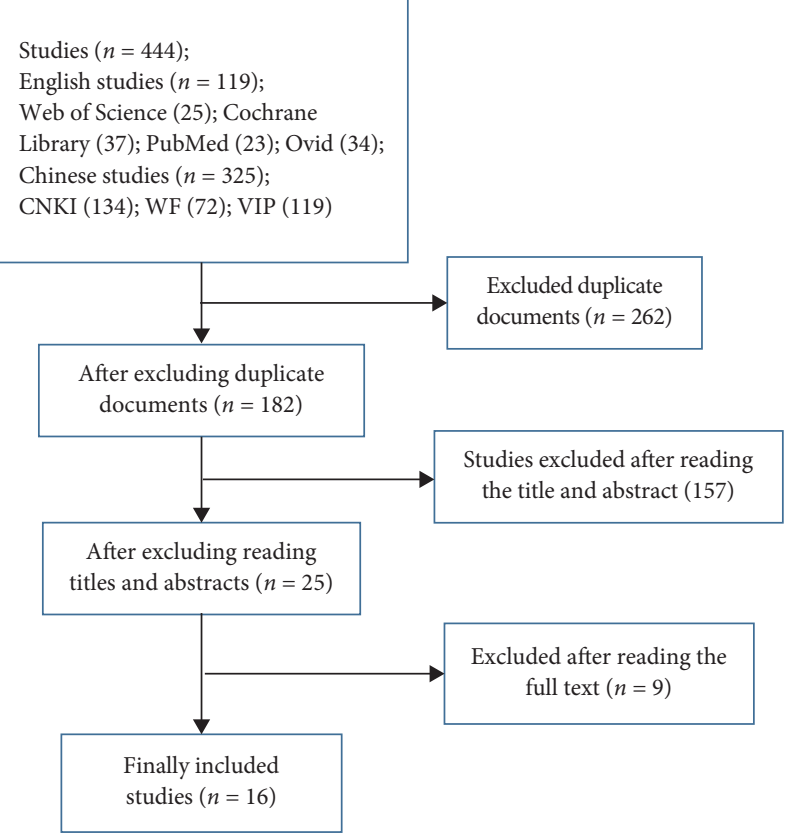

FIgURE 1: Flow chart of the study selection process.

\subsection{Outcome Analysis}

3.5.1. CARS Analysis. A total of 723 participants were included in nine studies $[21,23-26,28-31], I^{2}=96 \%$. Heterogeneity existed. We performed a subgroup analysis. According to the intervention factors of the experimental group, the group was divided into two subgroups, namely, acupuncture and acupuncture plus massage $[23,28]$. For the acupuncture subgroup, $P=0.10$ and $I^{2}=43 \%$. Thus, we selected the fixed-effect model $(\mathrm{MD}=-2.65,95 \% \mathrm{CI}(-3.22$, $-2.07)$, $(P<0.00001))$. For the acupuncture plus massage subgroup, $P=0.32$ and $I^{2}=0 \%$. Thus, we used a fixed-effect model $\quad(\mathrm{MD}=-10.35, \quad 95 \% \quad$ CI $\quad(-11.34, \quad-9.36)$ $(P<0.00001))$. The analysis results of both subgroups were statistically significant, as shown in Figure 4.

3.5.2. ABC Analysis. A total of 819 participants were included in 10 studies [17, 21, 23, 25, 26, 28, 29, 31-33]. Results showed that heterogeneity $\left(I^{2}=93 \%\right)$ existed. Through subgroup and sensitivity analyses, no significant change in heterogeneity was found. We selected the random-effect model $(\mathrm{MD}=-6.70,95 \%$ CI $(-9.10,-4.29), P<0.00001)$. The difference between the two groups was found to be statistically significant, as shown in Figure 5.

3.5.3. Analysis of $A B C$ 's Subitems. A total of 211 participants were included in three studies [21-24, 27]. The analysis results of sensory, relating, language, body and object use, and social/self-help in the subitems of ABC showed heterogeneity as follows: $I^{2}=88 \%, I^{2}=75 \%, I^{2}=93 \%, I^{2}=83 \%$, and $I^{2}=66 \%$, respectively. Further sensitivity analysis revealed that the control group in literature (Zhang Huichun, 2019) used the Taiwanese Kidd children's sensory integration training, which differs from two other studies in 


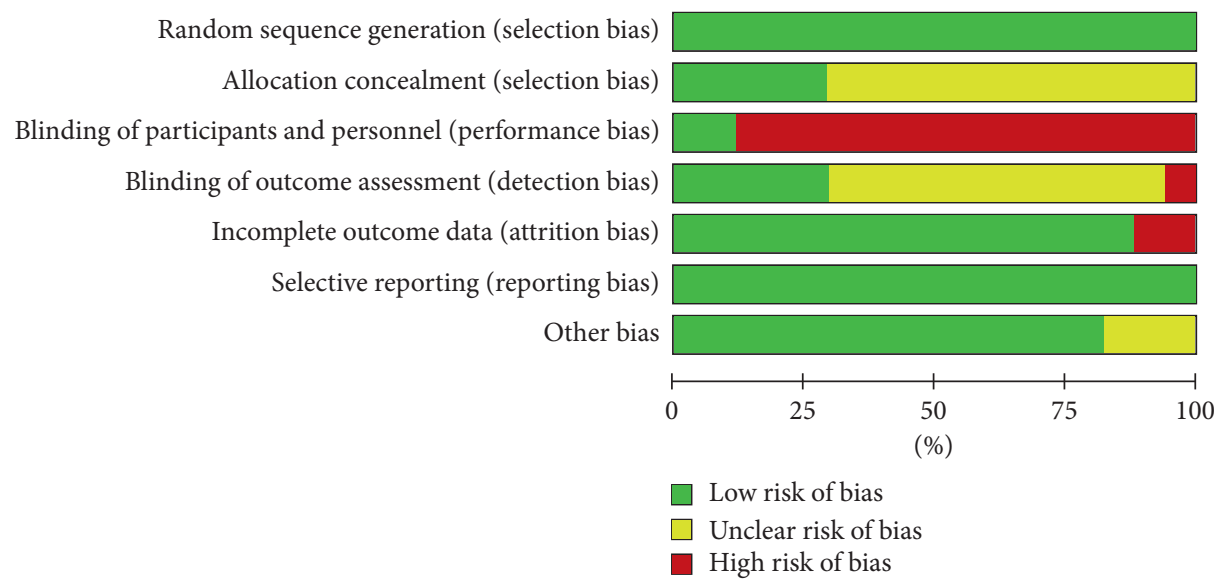

Figure 2: Risk of bias in included studies (A).

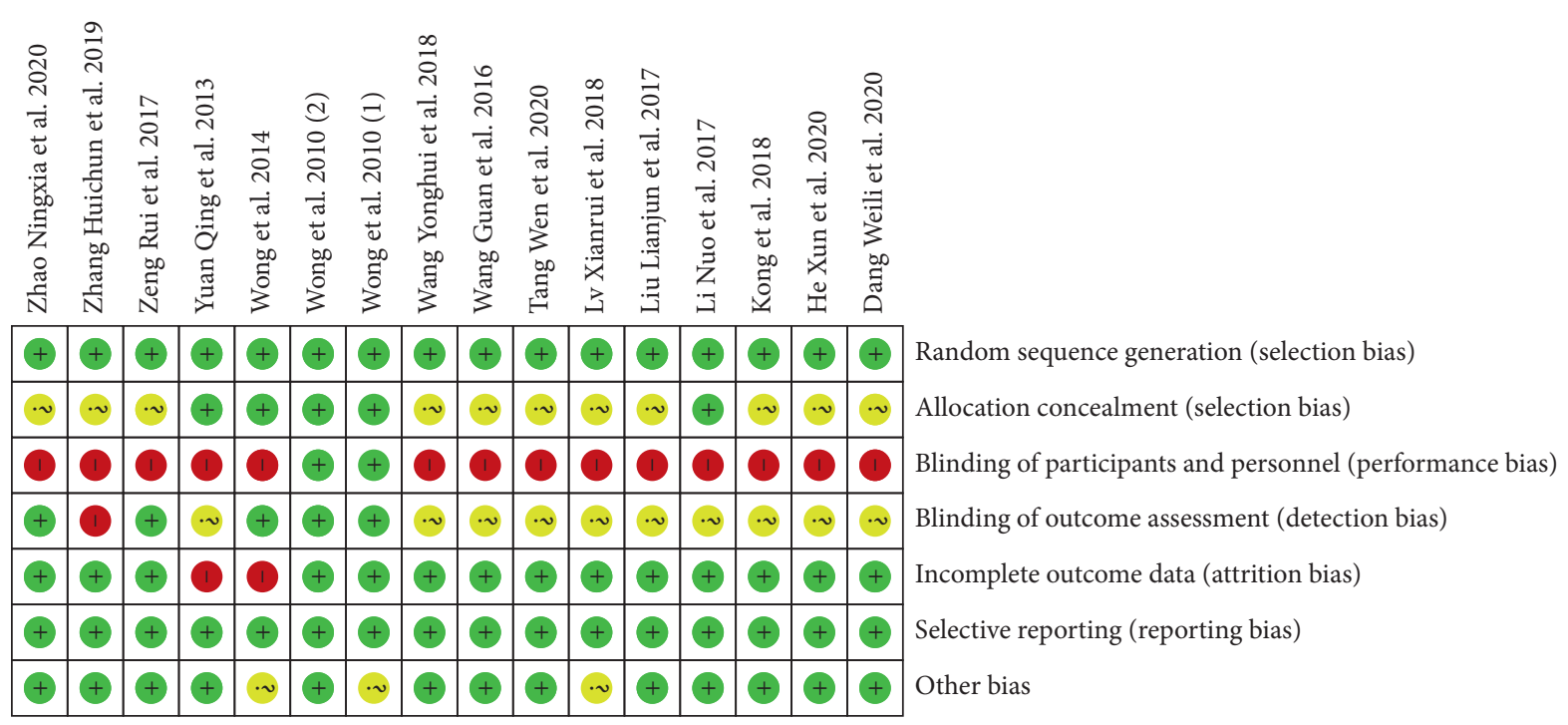

Figure 3: Risk of bias in included studies (B).

terms of conventional rehabilitation training [21]. This factor was analyzed as a possible cause of heterogeneity, and the analysis was performed after removing it. As shown in Figure 6, the fixed-effect model results were as follows: sensory $\left(P=0.29, I^{2}=10 \%\right),(\mathrm{MD}=-2.67,95 \% \mathrm{CI}(-2.90$, $-2.44), \quad P<0.00001) \quad($ Figure $6(\mathrm{a}))$; relating $(P=0.18$; $\left.I^{2}=46 \%\right),(\mathrm{MD}=-3.28,95 \%$ CI $(-3.55,-3.02), P<0.00001)$ (Figure 6(b)); language $\left(P=0.73, I^{2}=0 \%\right),(\mathrm{MD}=-2.45$, 95\% CI $(-2.73,-2.16), P<0.00001)$ (Figure 6(c)); body and object use $\left(P=0.53 ; I^{2}=0 \%\right),(\mathrm{MD}=-1.19,95 \%$ CI $(-1.38$, -1.00), $\quad P<0.00001$ ) (Figure $6(\mathrm{~d})$ ); and social/self-help $\left(P=0.43 ; I^{2}=0 \%\right),(\mathrm{MD}=-2.09,95 \%$ CI $(-2.30,-1.89)$, $P<0.00001$ ) (Figure 6(e)).

3.5.4. RDLS Analysis. A total of 126 participants were included in three studies [17-19]. Figure 7 presents the analysis results of comprehension and expression ages in the subitems of RDLS. The results for comprehension age were as follows: $\left(P=0.24 ; I^{2}=30 \%\right)$, select fixed-effect model, $(\mathrm{MD}=0.08,95 \% \mathrm{CI}(-0.06,0.22), P=0.27)$, and the two groups were statistically significant (Figure $7(\mathrm{a})$ ). The results for expression age were as follows: $\left(P=0.47 ; I^{2}=0 \%\right)$, select the fixed-effect model, $(\mathrm{MD}=0.15,95 \% \mathrm{CI}(0.04,0.26)$, $P=0.009$ ), and the two groups were statistically significant (Figure 7(b)).

3.5.5. WeeFIM Analysis. A total of 126 participants were included in three studies [17-19]. The analysis results were as follows: $\left(P=0.15 ; I^{2}=48 \%\right)$, select fixed-effect model, $(\mathrm{MD}=3.70,95 \% \mathrm{CI}(2.38,5.02), P<0.00001)$, and the two groups were statistically significant (Figure 8 ).

3.5.6. Publication Bias. Among the observed outcome indicators, only $\mathrm{ABC}$ included 10 studies, and the other outcome indicators included in the study were $<10$. A funnel chart analysis was performed on ABC. Figure 9 shows that the distribution of the funnel graph was asymmetric, and a certain degree of bias was present. The following problems existed in the analysis of the literature: the sample size of the included studies was generally low, the risk of publication 


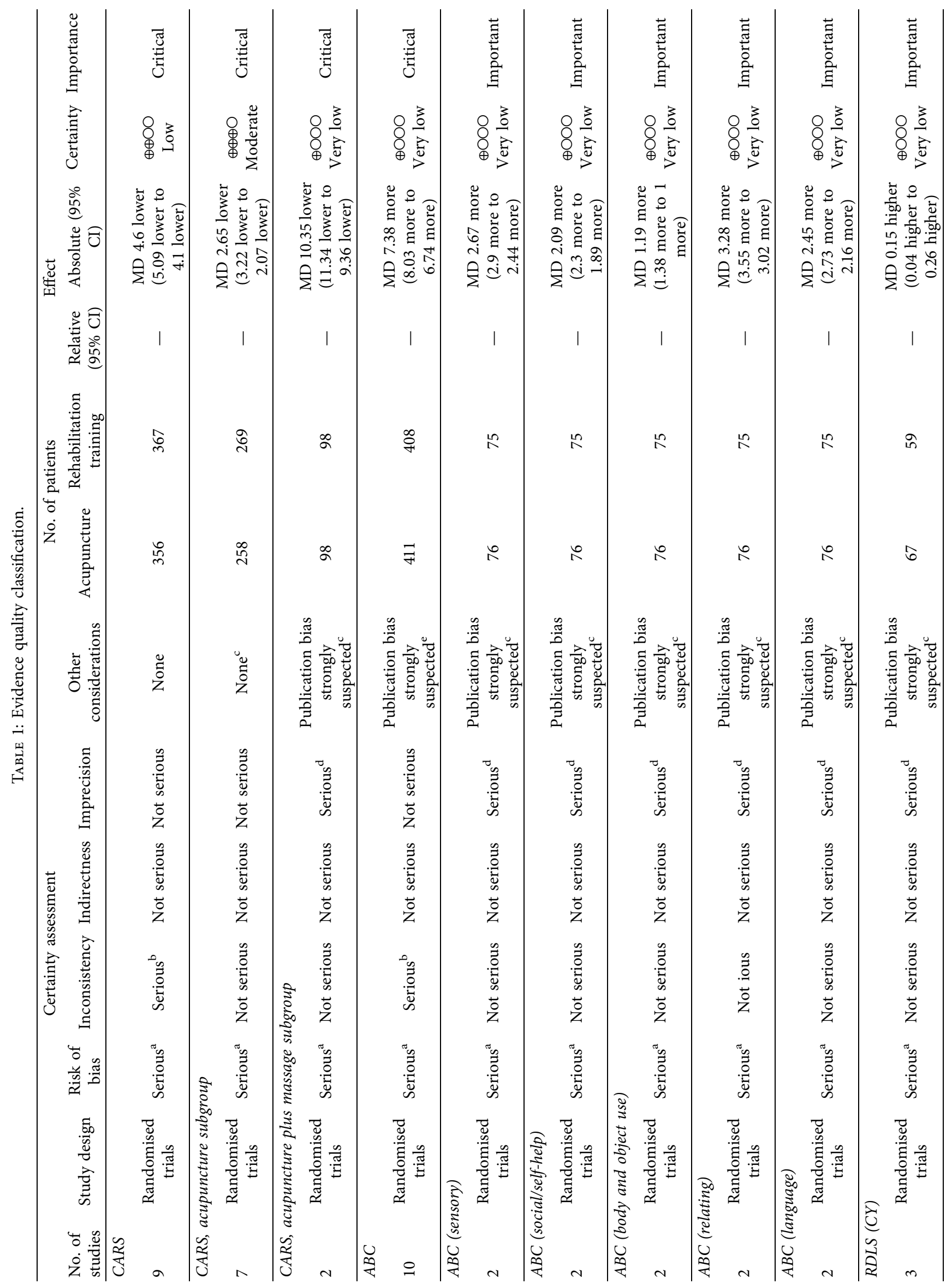




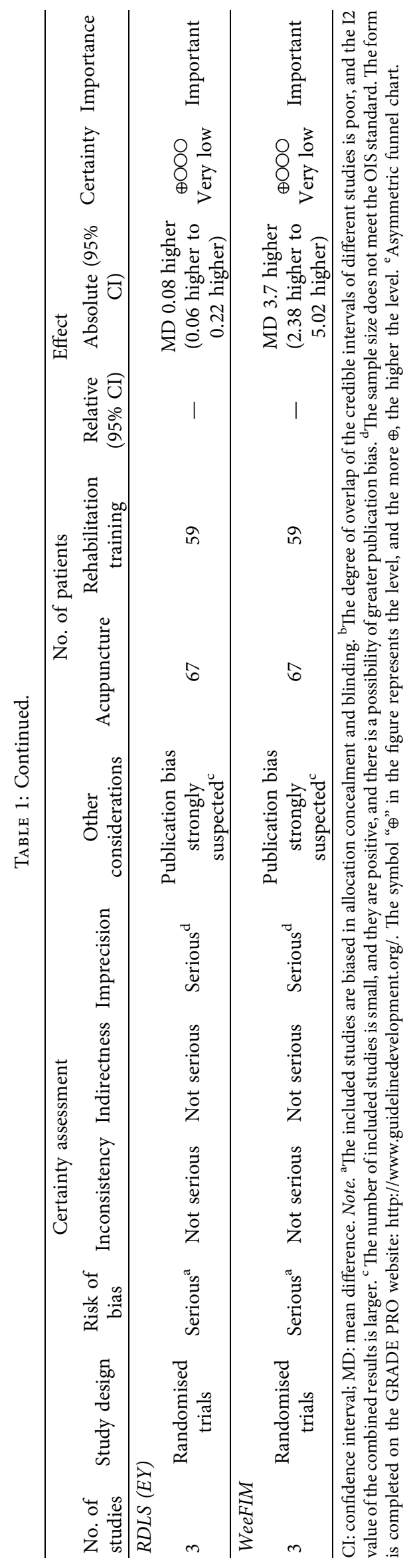




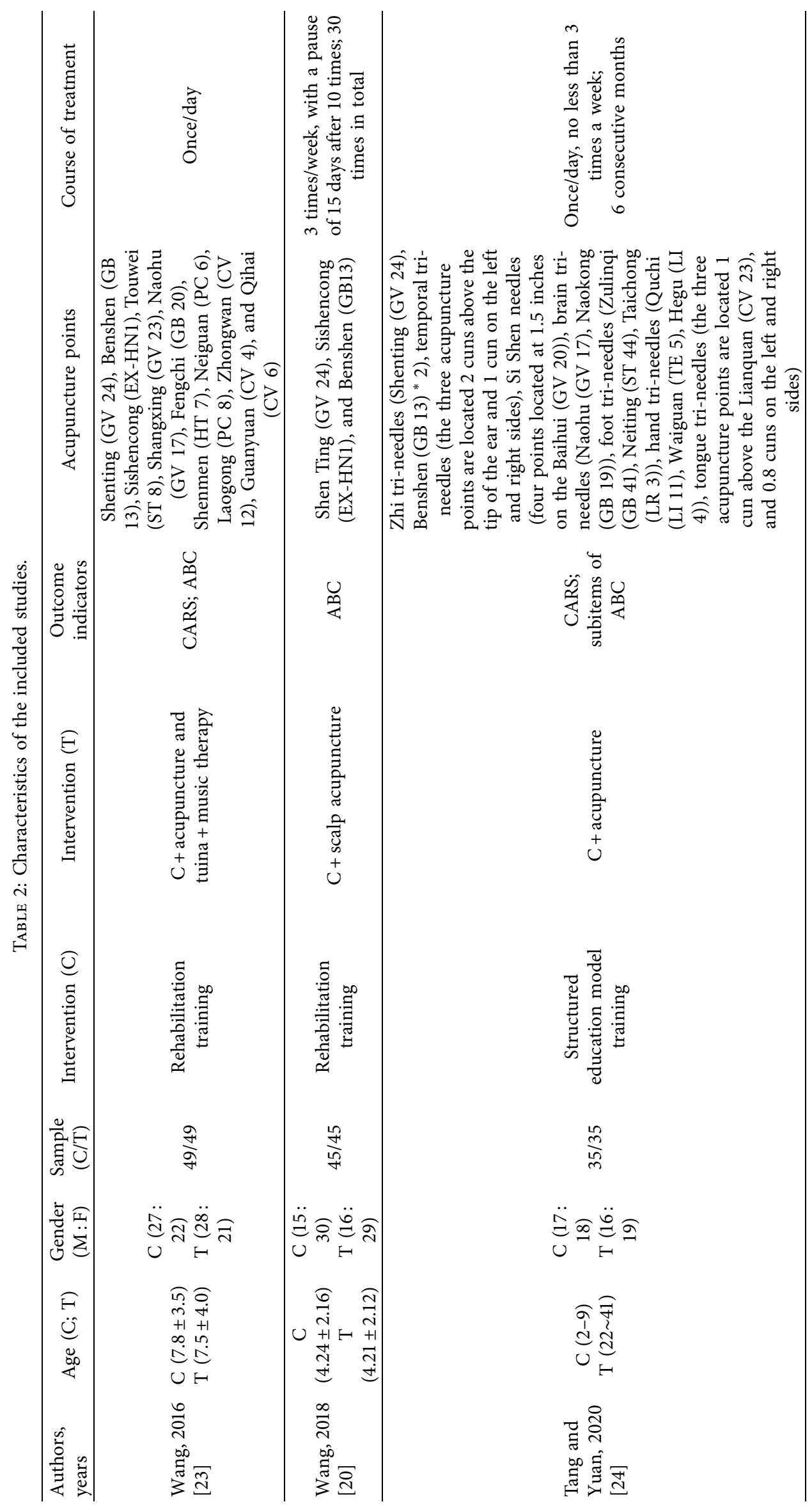




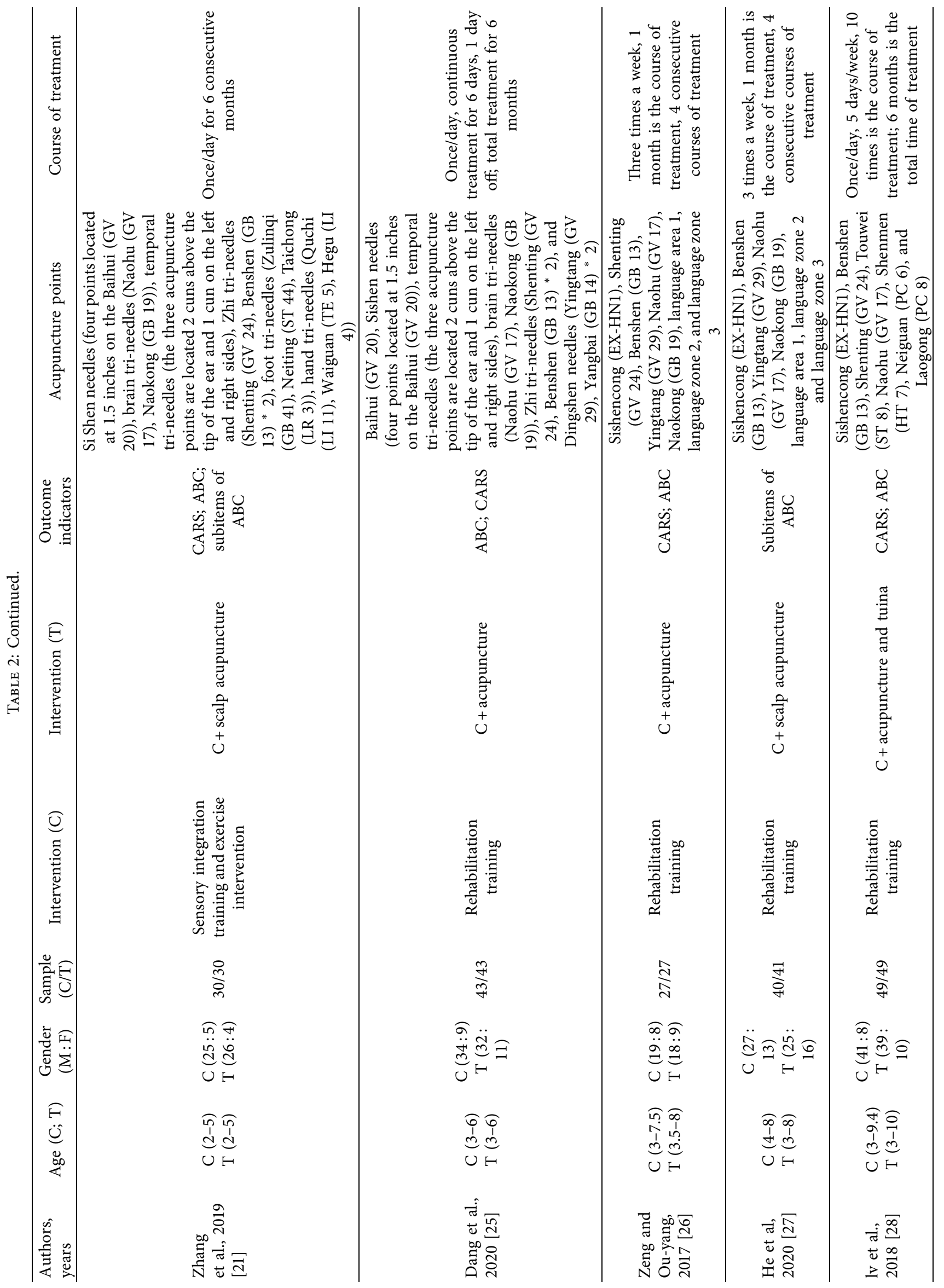




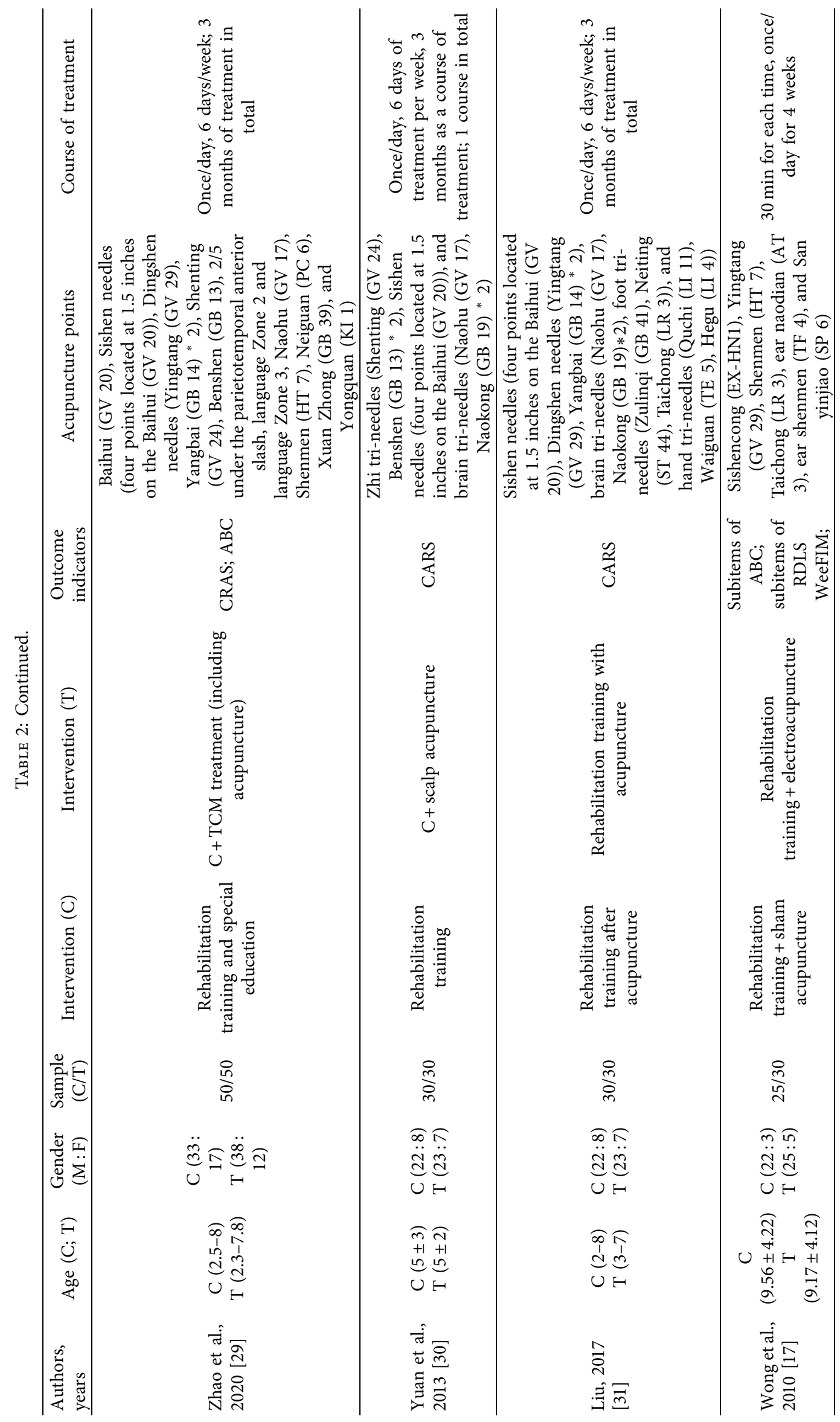




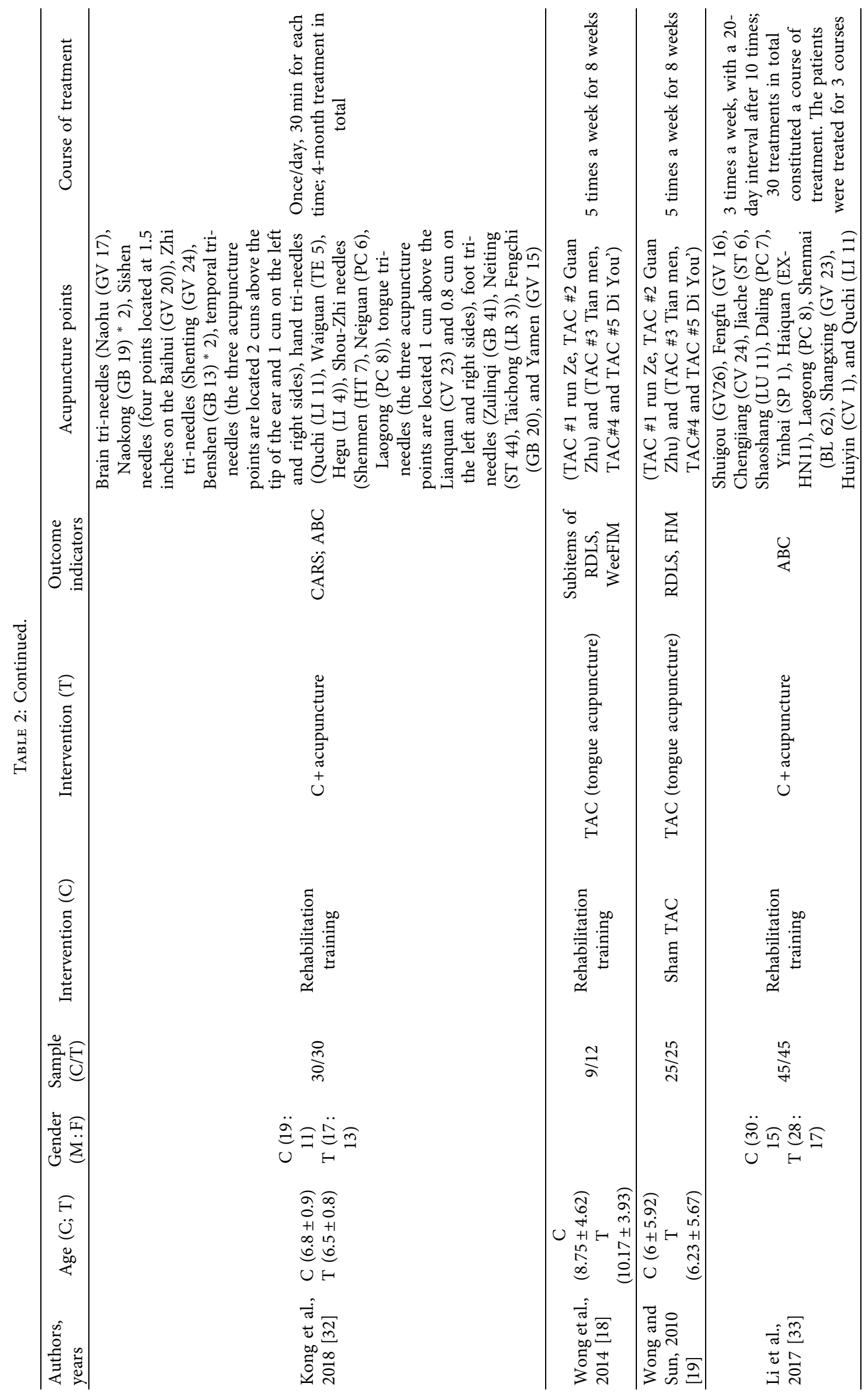




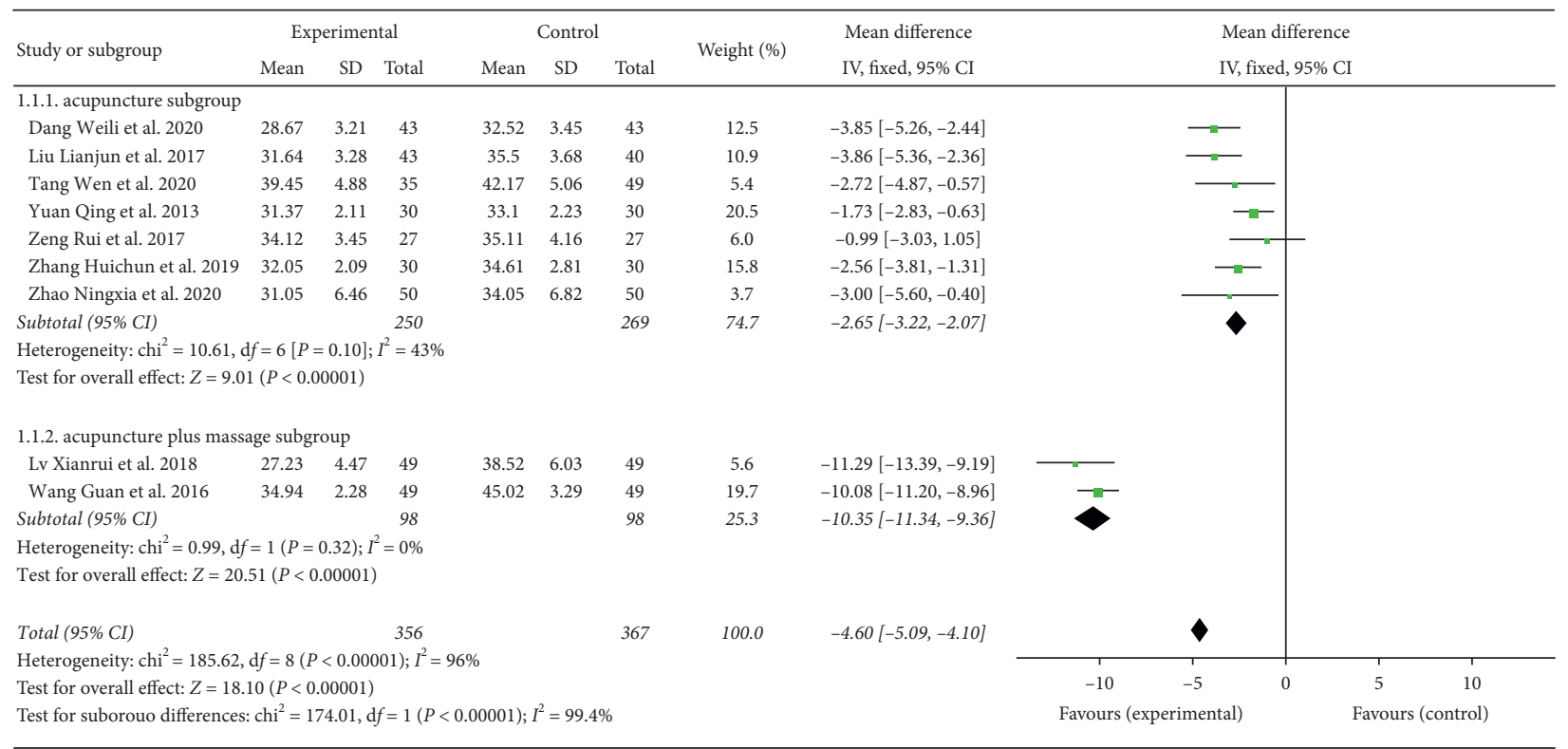

FIGURE 4: Forest plot showing MD (with 95\% CI) for CARS of included studies comparing the experimental group with the control group. Childhood autism rating scale (CARS).

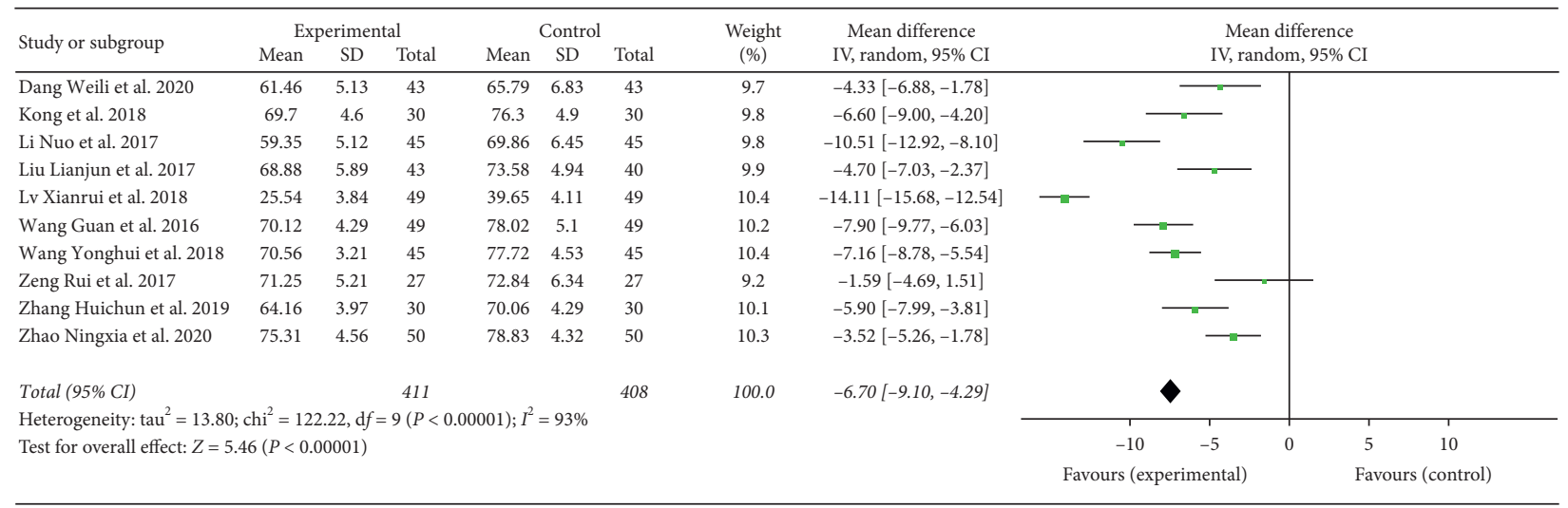

FIGURE 5: Forest plot showing MD (with 95\% CI) for ABC of included studies comparing the experimental group with the control group. $\mathrm{ABC}$ : autism behavior checklist.

bias in small randomised controlled trials may be high, and the included literature was only in Chinese and English, thereby excluding the literature in other languages. All of the above factors may cause publication deviation, as described in detail in Table 2.

3.5.7. Safety. Only four studies [17-19, 25] reported the safety of acupuncture for the treatment of ASD. A study [25] reported the presence of a subcutaneous bruise at the puncture site. The symptoms were mild and did not affect the treatment course. The remaining three studies [17-19] reported that some children experienced minor superficial bleeding or cried and showed irritability during acupuncture. The other included studies did not report safety issues.

\section{Discussion}

This review included 16 studies, which included 1332 patients. The experimental group had statistical differences in the scores of CARS, RDLS, WeeFIM, and ABC, and the subitems were compared with the control group. Among the 16 studies, two studies $[17,19]$ compared acupuncture and sham acupuncture. Results of analysis confirmed the effectiveness of acupuncture in the treatment of ADS. The other studies on the two groups' intervention methods 


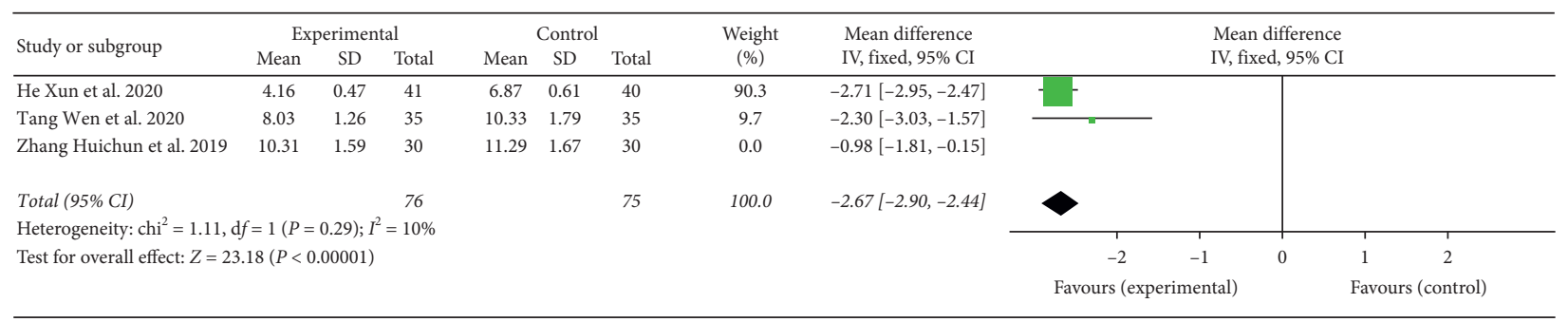

(a)

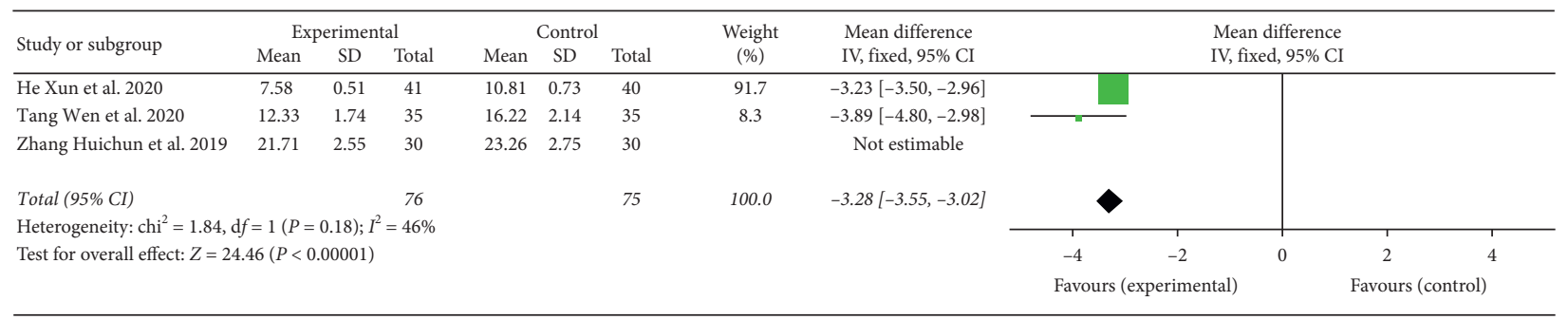

(b)

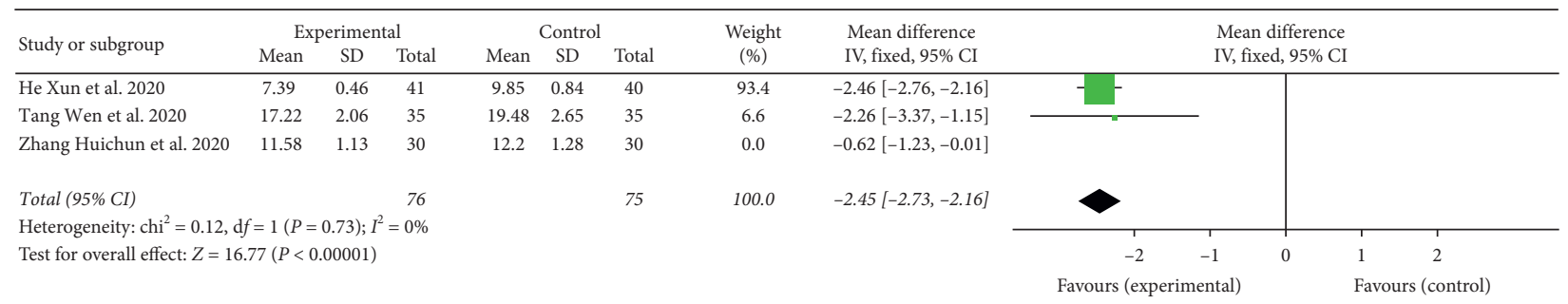

(c)

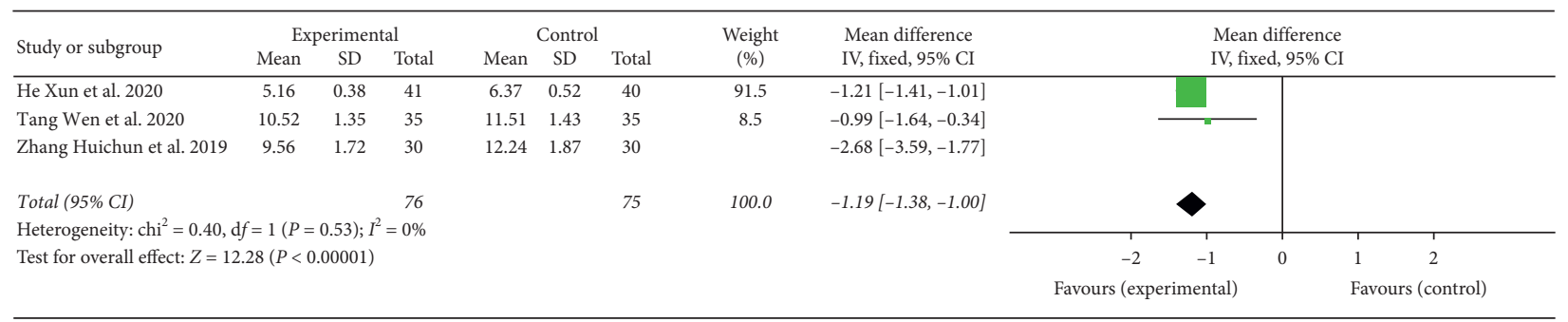

(d)

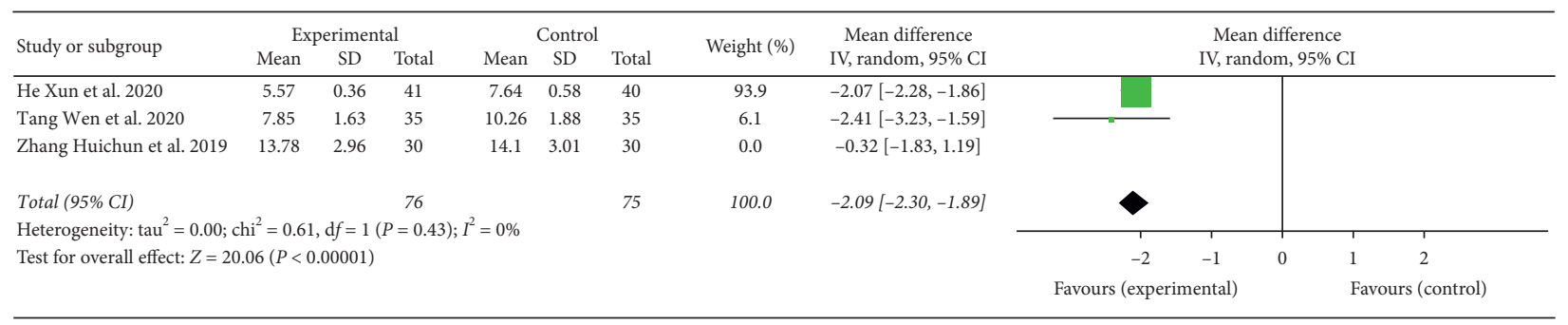

(e)

FIGURE 6: Forest plot showing MD (with 95\% CI) for subitems of autism behavior check list (ABC) of included studies comparing the experimental group with the control group. $\mathrm{ABC}$, subitems of $\mathrm{ABC}$ included sensory, relating, language, body and object use, and social/selfhelp. (a) Sensory, (b) relating, (c) language, (d) body and object use, and (e) social/self-help.

compared rehabilitation treatment and rehabilitation treatment plus acupuncture. Results suggested that the combination of acupuncture with other treatments improved the treatment effect. The findings suggested that acupuncture alleviated the various symptoms of ASD.
Acupuncture differs from the modern medicine theory, which states that autism is caused by brain dysfunction. In TCM, autism is believed to be mostly caused by congenital insufficiency, which leads to an imbalance in body function. The cause lies in the brain and is closely related to the heart, 


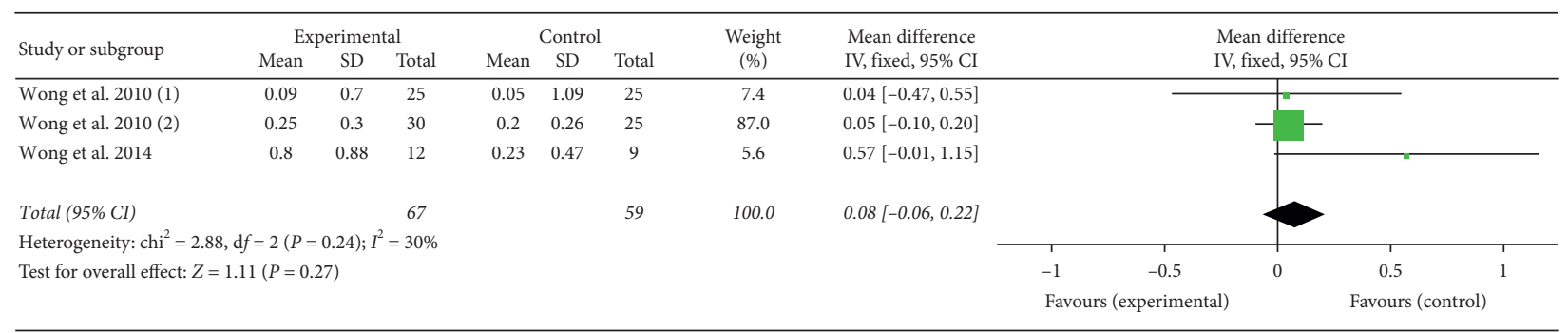

(a)

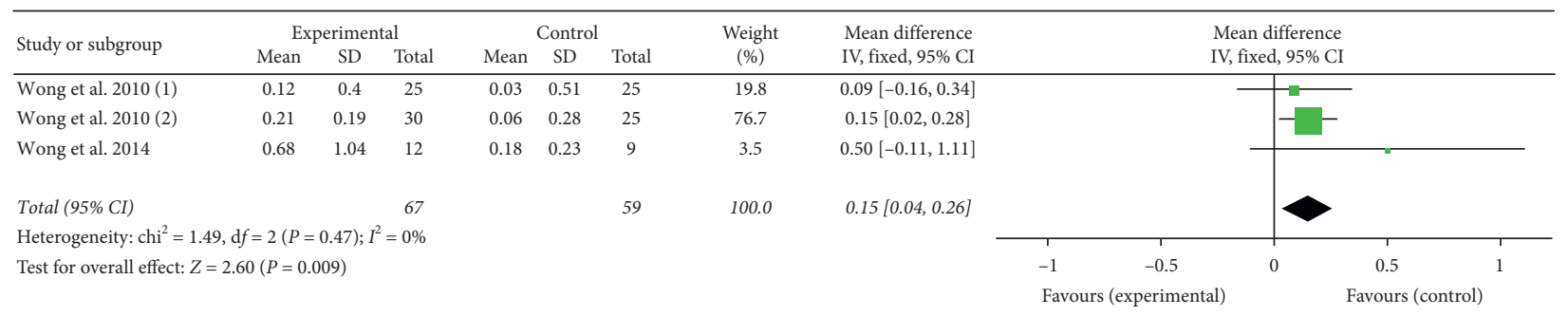

(b)

Figure 7: Forest plot showing MD (with 95\% CI) for subitems of Reynell developmental language scale (RDLS) of included studies comparing the experimental group with the control group. Subitems of RDLS included comprehension and expression ages. (a) Comprehension age; (b) expression age.

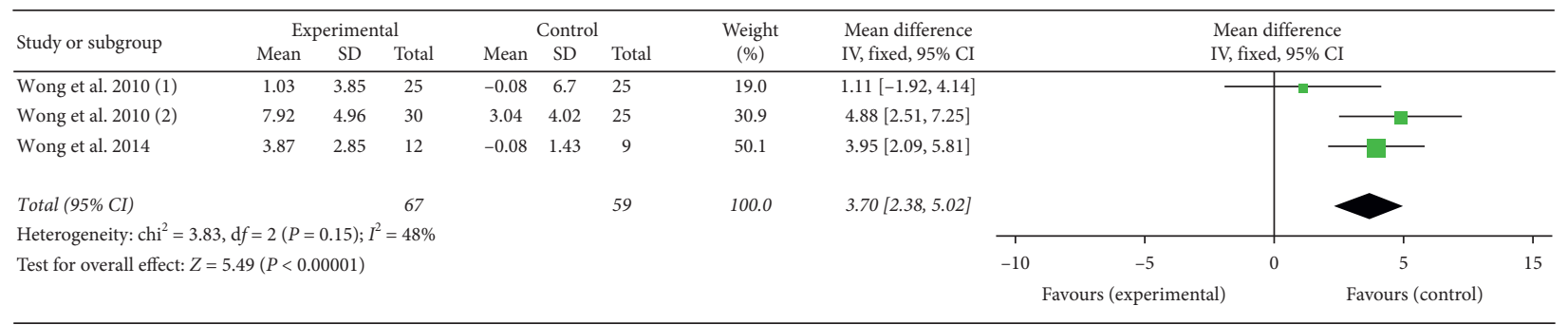

FIGURE 8: Forest plot showing MD (with 95\% CI) for WeeFIM of included studies comparing the experimental group with the control group. WeeFIM: functional independence measure of children.

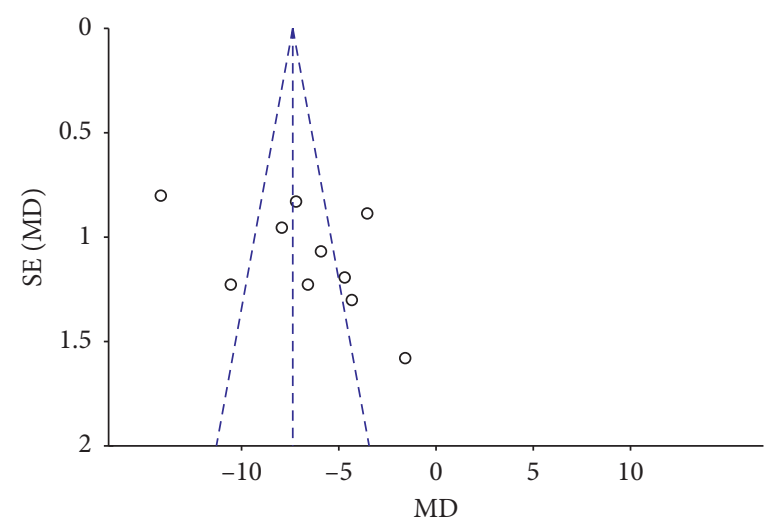

FIgURE 9: Funnel graph for ABC of included studies. ABC: autism behavior checklist.

liver, and kidneys [34]. Symptoms can be alleviated by applying acupuncture to relevant acupoints, that is, stimulating the head acupoints, namely, Si shencong (EX-HN1), Shenting (GV 24), Benshen (GB 13), Yintang (GV 29), Naohu (GV 17), and Naokong (GB 19), as well as other acupoints that can alleviate ASD. These acupoints are mostly distributed in the projection area of the frontal, parietal, and temporal lobes on the body surface. Stimulating these acupoints can adjust cortical function and brain electrical activity, thereby improving cerebral blood flow speed, 
promoting functional awakening and recovery, and improving intelligence. Acupuncture can alleviate affective disorder, attention disorder, and abnormal behavior [26]. Shenmen (HT 7) is one of the most commonly used acupoints in the acupuncture treatment of ASD. Wang Yu et al. found that acupuncture at Shenmen can regulate neurotransmitters, promote the secretion of neurotrophic factors, regulate the nerve-endocrine-immune network system, and inhibit cell apoptosis, thereby improving brain function [35]. Tongue acupuncture is one of the acupuncture methods $[18,19]$. According to TCM, the tongue is the intersection site of all 14 meridians. The physiological mechanism might be related to the fact that the tongue is close to the brainstem and cerebellum; thus, stimulating tongue acupoints might augment the neural pathways connected to the motor-somatosensory cortex [17]. In a randomised controlled trial that used a positron emission tomography scan of the brain as one of the outcome measures for acupuncture versus no acupuncture, results showed that acupuncture alleviated some core features of ASD [17].

Sixteen studies were finally included, and all of them reported the source of random assignment sequence. Only five studies [17-19, 30, 33] described allocation hiding. The remaining studies did not provide specific descriptions. Given that acupuncture and rehabilitation cannot guarantee blindness, only two studies [17, 19] implemented double blindness, and both groups were assigned a control group that was treated with sham acupuncture. Five studies $[17-19,26,29]$ reported blinding in the evaluation of experimental results. One study [21] reported that the outcome evaluation was not blinded, whereas the remaining studies did not specify it. In summary, a certain risk of bias exists in the study-quality evaluation results. Results suggested that clinical trials and reports are insufficiently clear and precise.

In conclusion, acupuncture was found to be effective and safe in the treatment of ASD. However, the use of GRADEpro GDT online tool to classify the quality of evidence for the study indicated that the quality of most outcome indicators were low and very low. Only the acupuncture subgroup of CARS showed moderate quality. The conclusion of the study was very likely to be very different from the real situation. The most degraded factor was the limitation of the study, which showed that the randomised controlled trials included in the study had a large bias in method design, as reflected in the insufficient or unreasonable implementation of blinding and allocation concealment. In addition, the study also had high risks in terms of heterogeneity, accuracy, and publication bias.

In the literature, the acupuncture types and methods were relatively diverse. According to the form of acupuncture, some studies used medical staff to stimulate acupuncture points through manual manipulation $[25,36]$. Some studies used electric current to stimulate acupuncture points; electrical devices were used to perform acupuncture [17, 37]. Acupuncture was classified according to the body part treated as follows: body acupuncture [17], head acupuncture [31], latch acupuncture [18, 19], and so on. Additionally, under the guidance of the TCM theory, different acupuncture points were selected based on changes in different symptoms and syndrome types. Existing research shows no standardized and unified treatment plan for the acupuncture treatment of autism, and the treatment prescription required further standardization.

In the included literature, only one study [17] reported the results of follow-up and patient compliance. Four studies [17-19, 25] reported side effects. No obvious side effects were reported in the results. A study by Dang Weili et al. [25] reported a subcutaneous bruise at the puncture site. The symptoms were mild and did not affect the treatment course. The remaining three studies [17-19] reported that some of the children experienced minor superficial bleeding and cried or showed irritability during acupuncture. Most children easily adapted and tolerated this technology. The compliance of children with autism is a problem in the treatment process, and for acupuncture method, the compliance of children is expected to be difficult. However, most of the included studies did not report compliance problems and low-compliance solutions for children.

In summary, acupuncture exerted a certain curative effect on ASD and could alleviate ASD's core symptoms. However, acupuncture methods and prescriptions at this stage remain heterogeneous. Large sample size, rigorous research, and long observation and follow-up periods are required to prove the advantages of acupuncture's curative effect on ASD. In addition, studies need to use rigorous and standard research designs for further study to draw stronger conclusions about the advantages of the use of acupuncture to treat children with ASD.

\section{Data Availability}

The datasets used and analyzed in the current study are included within this article.

\section{Conflicts of Interest}

All authors declare there are no potential conflicts of interest with respect to the research, authorship, and/or publication of this article.

\section{Authors' Contributions}

W. L. and P. JL. designed the test plan, data sorting, and analysis, Q. FQ, C. WM, L. GW, Z. Y, G. TG, S. Y, and T. WZ assisted in sorting the data, and W. P. guided the test process. All authors have read and approved the final manuscript.

\section{References}

[1] "Guidelines for diagnosis, treatment and rehabilitation of childhood autism (health affairs office of the medical affairs Bureau [2010] no. 123)," Chinese Journal of Child Health Care, vol. 19, no. 03, pp. 289-294, 2011.

[2] J. Chen, "The trends in the development of autism diagnosis and service- - a prospect of the fifth edition of the draft "diagnostic and statistical manual of mental disorders," Chinese Journal of Special Education, vol. 8, pp. 59-65, 2011. 
[3] M. Elsabbagh, G. Divan, Y.-J. Koh et al., "Global prevalence of autism and other pervasive developmental disorders," Autism Research, vol. 5, no. 3, pp. 160-179, 2012.

[4] World Health Organization (WHO), Autism Spectrum Disorders: Fact Sheet, World Health Organization (WHO), Geneva, Switzerland, 2017.

[5] T. A. Lavelle, M. C. Weinstein, J. P. Newhouse, K. Munir, K. A. Kuhlthau, and L. A. Prosser, "Economic burden of childhood autism spectrum disorders," Pediatrics, vol. 133, no. 3, pp. e520-e529, 2014.

[6] T. T. Shimabukuro, S. D. Grosse, and C. Rice, "Medical expenditures for children with an autism spectrum disorder in a privately insured population," Journal of Autism and Developmental Disorders, vol. 38, no. 3, pp. 546-552, 2008.

[7] J. Brosnan and O. Healy, "A review of behavioral interventions for the treatment of aggression in individuals with developmental disabilities," Research in Developmental Disabilities, vol. 32, no. 2, pp. 437-446, 2011.

[8] B. X. Jin, J. L. Li, and Z. H. Liu, "Treatment of autism with scalp acupuncture," Zhong Guo Zhen Jiu, vol. 31, pp. 692-696, 2011.

[9] P. A. Rao and R. J. Landa, "Association between severity of behavioral phenotype and comorbid attention deficit hyperactivity disorder symptoms in children with autism spectrum disorders," Autism, vol. 18, no. 3, pp. 272-280, 2014.

[10] L. R. Warren, P. A. Rao, and D. C. Paton, "A pilot observational study of an acupressure/acupuncture intervention in children with autism spectrum disorder," Journal of Alternative and Complementary Medicine (New York, NY), vol. 23, pp. 1-8, 2017.

[11] I. A. Surapaty, C. Simadibrata, E. S. Rejeki, and I. Mangunatmadja, "Laser acupuncture effects on speech and social interaction in patients with autism spectrum disorder," Medical Acupuncture, vol. 32, no. 5, pp. 300-309, 2020.

[12] H. Won, W. Mah, and E. Kim, "Autism spectrum disorder causes, mechanisms, and treatments: focus on neuronal synapses," Frontiers in Molecular Neuroscience, vol. 6, no. 19, pp. 1-26, 2013.

[13] J. T. Mccracken, J. Mcgough, B. Shah et al., "Risperidone in children with autism and serious behavioral problems," New England Journal of Medicine, vol. 347, no. 5, pp. 314-321, 2002.

[14] C. Liu, T. Li, Z. Wang et al., "Scalp acupuncture treatment for children's autism spectrum disorders," Medicine, vol. 98, no. 13, pp. 1-8, 2019.

[15] S. E. Levy and S. L. Hyman, "Complementary and alternative medicine treatments for children with autism spectrum disorders," Child \& Adolescent Psychiatric Clinics of North America, vol. 24, pp. 117-143, 2015.

[16] V. C. N. Wong, "Use of complementary and alternative medicine (CAM) in autism spectrum disorder (ASD): comparison of Chinese and western culture (part A)," Journal of Autism and Developmental Disorders, vol. 39, no. 3, pp. 454-463, 2009.

[17] V. Wong, W. X. Chen, and W. L. Liu, "Randomized controlled trial of electro-acupuncture for autism spectrum disorder," Alternative Medicine Review, vol. 15, no. 2, pp. 136-146, 2010.

[18] V. C.-N. Wong, J.-G. Sun, and D. W.-C. Yeung, "Randomized control trial of using tongue acupuncture in autism spectrum disorder," Journal of Traditional Chinese Medical Sciences, vol. 1, no. 1, pp. 62-72, 2014.

[19] V. C.-N. Wong and J.-G. Sun, "Randomized controlled trial of acupuncture versus sham acupuncture in autism spectrum disorder," The Journal of Alternative and Complementary Medicine, vol. 16, no. 5, pp. 545-553, 2010.

[20] Y. Wang, "Effect of acupuncture and moxibustion on improving language and communication ability of children with autism," Chronic Pathematology Journal, vol. 19, no. S1, pp. 61-62, 2018.

[21] H. Zhang, Q. Shang, C. Ma et al., "Effects of acupuncture combined with sensory integration training and exercise intervention on autistic children," Journal of Clinical and Pathological Research, vol. 39, no. 2, pp. 371-376, 2019.

[22] M. Choi, S. H. Lee, and G. T. Chang, "Herbal medicine treatment for influenza: a systematic review and meta-analysis of randomized controlled trials," The American Journal of Chinese Medicine, vol. 48, no. 7, pp. 1553-1576, 2020.

[23] G. Wang, "Intervention on the rehabilitation of children autism treated with acupuncture, Tuina and TCM five-element theory," World Journal of Integrated Traditional and Western Medicine, vol. 11, no. 7, pp. 968-971, 2016.

[24] W. Tang and X. Yuan, "Analysis of the effect of acupuncture combined with structured education mode in the treatment of children with autism," Journal of Qiqihar Medical University, vol. 41, no. 12, pp. 1505-1507, 2020.

[25] W. Dang, W. Li, and B. Ma, "Effects of acupuncture intervention on core symptoms in children with autism spectrum disorder," Chinese Journal of Rehabilitation Medicine, vol. 35, no. 5, pp. 527-532, 2020.

[26] R. Zeng and B.-S. Ouyang, "Clinical research on scalp acupuncture combined with rehabilitation training for autism children," Journal of Clinical Acupuncture and Moxibustion, vol. 33, no. 1, pp. 18-20, 2017.

[27] X. He and Y. Meng, "Effect of scalp acupuncture combined with rehabilitation training on behavioral function of children with autism," Forum on Traditional Chinese Medicine, vol. 35, no. 3, pp. 44-46, 2020.

[28] X. Lv, H. Fan, Q. Li et al., "Clinical study of massage combined acupuncture and moxibustion on autistic children based on the five elements theory of TCM," World Chinese Medicine, vol. 13, no. 7, pp. 1731-1735, 2018.

[29] N. Zhao, X. Du, H. Song et al., "Application of traditional Chinese medicine clinical pathway for children with autism spectrum disorder," Chinese Journal of Medicinal Guide, vol. 22, no. 5, pp. 305-309, 2020.

[30] Q. Yuan, Y. Liu, Y. Yu et al., "Treatment of autism children: observation on efficacy of behavior training with retention of needles on head," Chinese Journal of Acupuncture and Moxibustion, vol. 33, no. 7, pp. 609-613, 2013.

[31] L. Liu, "Clinical study on interactive scalp acupuncture therapy for autism spectrum disorders," Shang Hai Journal of Acupuncture and Moxibustion, vol. 36, no. 11, pp. 1303-1306, 2017.

[32] F. S. Kong, D. Hu, Q. Yuan et al., "Efficacy of acupuncture on children with autism spectrum disorder," International Journal of Clinical and Experimental Medicine, vol. 11, no. 12, pp. 13775-13780, 2018.

[33] N. Li, J.-L. Li, Z.-H. Liu et al., "Clinical observation on acupuncture at thirteen ghost acupoints for children with autism spectrum disorder," Journal of Acupuncture and Tuina Science, vol. 15, no. 5, pp. 344-348, 2017.

[34] G. Liu and L. Yuan, "Analysis on the etiology, pathogenesis and syndrome differentiation of children with autism in traditional Chinese medicine," Liaoning Journal of Traditional Chinese Medicine, vol. 9, pp. 1226-1227, 2007.

[35] Y. Wang and X. Lin, "Research progress of effect of acupuncture Shenmen acupoint on the brain function," Clinical 
Journal of Traditional Chinese Medicine, vol. 29, no. 10, pp. 1774-1776, 2017.

[36] G. Liu and L. Yuan, "Clinical observe of acupuncture treat autism," Chinese Archives of Traditional Chinese Medicine, vol. 25, no. 12, pp. 2497-2498, 2007.

[37] Q. Yuan, Z. Wu, R. Wang et al., "Observation on the therapeutic effect of acupuncture treatment of autism children," Acupuncture Research, vol. 34, no. 3, pp. 183-187, 2009. 